\title{
Restauration d'une icône-calendrier russe du musée du Temps de Besançon
}

\section{Aline Berelowitsch}

\section{(2) OpenEdition}

1 Journals

Édition électronique

URL : http://journals.openedition.org/imagesrevues/864

DOI : 10.4000/imagesrevues.864

ISSN : $1778-380$

Éditeur :

Centre d'Histoire et Théorie des Arts, Groupe d'Anthropologie Historique de l'Occident Médiéval, Laboratoire d'Anthropologie Sociale, UMR 8210 Anthropologie et Histoire des Mondes Antiques

\section{Référence électronique}

Aline Berelowitsch, "Restauration d'une icône-calendrier russe du musée du Temps de Besançon », Images Re-vues [En ligne], Hors-série 1 | 2008, mis en ligne le 15 mars 2011, consulté le 30 janvier 2021. URL : http://journals.openedition.org/imagesrevues/864 ; DOI : https://doi.org/10.4000/ imagesrevues.864

Ce document a été généré automatiquement le 30 janvier 2021.

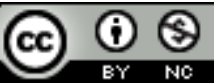

Images Re-vues est mise à disposition selon les termes de la Licence Creative Commons Attribution Pas d'Utilisation Commerciale 4.0 International. 


\title{
Restauration d'une icône-calendrier russe du musée du Temps de Besançon $^{1}$
}

\author{
Aline Berelowitsch
}

1 Le musée du Temps de Besançon conserve une icône-calendrier ${ }^{2}$, mesurant $45 \times 24 \mathrm{~cm}$, qui est peinte selon la technique traditionnelle de la tempéra, c'est-à-dire avec des pigments et un liant à l'œuf, sur un panneau de tilleul de 2,6 cm d'épaisseur, avec une préparation constituée de colle de peau et de sulfate de calcium. La surface du panneau est divisée en cinq registres horizontaux, eux-mêmes séparés en vignettes (fig.1). À chaque jour du mois correspond une vignette, où figure le saint ou la fête du jour. Le jour est signifié par une lettre rouge et les noms des saints sont inscrits en noir audessus de la vignette. L'icône est incomplète : en effet, elle était peinte à l'origine sur deux panneaux assemblés, mais le panneau de droite s'est décollé et a été perdu. 
Fig. 1

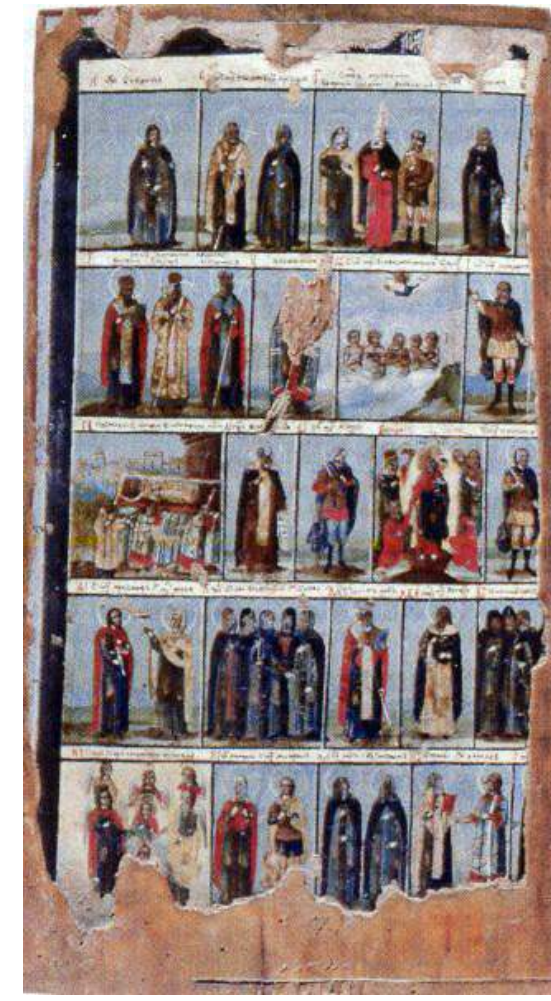

Vue d'ensemble de la face de l'œuvre après restauration

2 Elle a été obtenue, ainsi que l'indique une inscription au revers de l'œuvre, par le zouave Eugène Grenier qui se trouvait à Sébastopol à l'époque de la guerre de Crimée, en 1856, et provenait probablement de la cathédrale Saint-Vladimir, située dans le centre de Sébastopol, qui possédait une série de ménologes pour les douze mois de l'année. Il ne reste malheureusement plus trace des onze autres icônes de cet ensemble. Eugène Grenier l'a ensuite rapportée en France dans sa ville natale de Besançon. Il l'a offerte à un chanoine de son diocèse, qui en a lui-même fait don au musée des BeauxArts. Elle est entrée dans la collection du musée du Temps en 1997, quand elle a été identifiée comme une icône-calendrier.

3 Les icônes-calendriers ou ménologes sont apparues dans l'art byzantin dès le $\mathrm{XI}^{\mathrm{e}}$ siècle, et en Russie au XVI ${ }^{e}$ siècle. Elles trouvent leur origine dans les recueils enluminés de vies de saints byzantins, appelés également ménologes. Dans ces compilations hagiographiques, les notices sont arrangées selon la date de la célébration de chaque saint dans le calendrier religieux. Dès les premiers siècles de la religion chrétienne, les apôtres, les martyrs et les confesseurs font l'objet de dévotions particulières. Les commémorations de martyrs le jour de leur mort commencèrent à se développer à partir du III ${ }^{e}$ siècle. L'Église a ainsi investi le calendrier civil de ses propres fêtes et de son rythme liturgique. Le calendrier religieux se fixa petit à petit. À partir $\mathrm{du} \mathrm{Ix}^{\mathrm{e}}$ siècle, il était utilisé dans tout l'empire byzantin. Avec l'établissement du calendrier religieux de l'Église de Constantinople, chaque saint trouva sa place dans l'année liturgique et acquit en même temps une physionomie spécifique ${ }^{3}$.

4 À la fin du $\mathrm{X}^{\mathrm{e}}$ siècle, sous le règne de Basile II, un moine byzantin, Siméon Métaphraste, a revu et corrigé dans un style plus soutenu les vies de saints relatées dans les ménologes. Jusqu'alors, les ménologes comprenaient une sélection de vies de certains 
saints. Siméon Métaphraste a arrangé son ménologe de manière à faire correspondre une vie de saint à chaque jour de l'année liturgique. Chaque vie de saint a été illustrée d'une miniature représentant un portrait ou le martyre du saint ${ }^{4}$. Les textes étaient destinés à être lus à haute voix pendant les services du matin et du soir, le jour de la fête du saint. Son ménologe de cent cinquante textes, de septembre à août, a eu un grand succès. Il a remplacé tous les autres ménologes et son emploi régulier est attesté dans les monastères dès le XII ${ }^{e}$ siècle.

C'est à Byzance, au XI ${ }^{e}$ siècle, que les icônes ménologes font leur apparition. La surface du panneau de l'icône-ménologe est divisée en registres horizontaux, compartimentés en vignettes où sont représentées des figures de saints (portraits ou scènes de martyre) ou des fêtes religieuses, disposées selon l'ordre du calendrier religieux. Chaque saint porte au-dessus de lui le jour du mois et une inscription avec son nom. Les saints sont généralement représentés sur fond d'or. Dans la plupart des cas, les saints et les fêtes d'un seul mois sont rassemblés sur un panneau. Il existait donc des ensembles de douze icônes pour l'année, une pour chaque mois. Mais l'on trouve aussi des icônescalendriers sous forme de diptyque, triptyque ou tétraptyque, sur lesquelles se suivent les saints de plusieurs mois.

6 Ces icônes étaient exposées dans les églises afin que les fidèles puissent les consulter comme un calendrier. Elles servaient aussi à la méditation. Le fidèle pouvait se recueillir devant la figure du saint dont on célébrait la fête. Elles étaient disposées sur les colonnes de la nef, ou bien, chaque mois, l'icône correspondante était placée sur un pupitre devant l'autel et servait de support à la liturgie.

7 Les plus anciens exemples d'icônes-calendriers russes datent du XVI ${ }^{e}$ siècle, alors que l'Église de Russie développe sa propre littérature religieuse (vies de saints, textes liturgiques) et canonise un grand nombre de saints russes ${ }^{5}$. Les saints sont toujours représentés sur fond d'or, serrés les uns contre les autres, mais leurs physionomies diffèrent (on n'y trouve plus l'influence des canons classiques). Les scènes de martyre sont rares et le style se distingue par une plus grande sobriété. Les ménologes russes ne désavouent cependant pas les ménologes byzantins pour ce qui est de la richesse des détails, des couleurs et du soin apporté à l'exécution. En Russie, les ménologes connurent une très grande popularité à partir du XVIII ${ }^{\mathrm{e}}$ siècle et surtout au XIX $\mathrm{X}^{\mathrm{e}}$ siècle.

8 L'icône-calendrier du musée du Temps est l'une des rares œuvres de ce type dans les collections françaises. CEuvre modeste par rapport aux grands ménologes pour l'année ou aux précieux ménologes sur fond d'or, elle est un exemple du développement des icônes-calendriers au XIXe siècle en Russie. Elle se caractérise par une grande simplicité : peu de détails, une gamme de couleurs restreinte, des fonds unis avec une bande de couleur plus sombre pour indiquer le sol, quasiment pas d'architectures et un nombre de personnages réduit (dans les scènes de groupe). L'influence occidentale (qui apparaît au XVIII e siècle dans la peinture d'icônes) se manifeste dans la rondeur des visages modelés en tons roses plus ou moins soutenus et non plus à partir du ton ocrevert éclairci progressivement des icônes traditionnelles, et dans les plis plus mous, moins structurés que dans les icônes de facture classique. Elle témoigne, par son style et sa technique, des particularités d'un art de l'icône tardif et populaire'.

9 L'intervention majeure de restauration sur cette œuvre a consisté dans le dégagement de la couche de vernis oxydé qui recouvrait l'œuvre. La couche protectrice des icônes est traditionnellement constituée d'huile et de résine. Les analyses effectuées sur l'œuvre ont indiqué la présence d'huile, de colophane, résine bon marché et de 
mauvaise qualité employée sur les icônes à partir du XVIII ${ }^{e}$ siècle, ainsi que d'un siccatif au plomb. Le vernis de l'œuvre était très altéré : le brunissement très prononcé et le durcissement qu'il présentait sont typiques du vernis huileux des icônes. La couche sombre et opaque de vernis empêchait la transmission de l'image, en masquant les détails et modifiant la perception des couleurs. De plus, le vernis ne remplissait plus sa fonction de protection, en raison de l'acidité et du durcissement de la colophane ${ }^{7}$, et de la rétraction due à l'excès de siccatif.

Nous avons donc choisi de nettoyer l'icône, en employant une technique de dégagement mécanique sous loupe binoculaire que certains restaurateurs, notamment en Russie, ont commencé à employer dans les années 1970. Cette technique permet d'éviter les problèmes que peuvent causer les solvants employés pour dissoudre la couche de vernis huileux: la couche picturale mince des icônes russes est particulièrement vulnérable aux agressions chimiques et peut être ramollie en même temps que le vernis ${ }^{8}$. Les solvants peuvent altérer les glacis minces et les rehauts d'or, et, sans microscope, le restaurateur ne peut avoir une vision précise de la stratigraphie de l'œuvre. Le dégagement s'effectue à l'aide de scalpels dont l'extrémité est taillée en forme de biseau. La partie tranchante est très étroite, large d'environ $1,5 \mathrm{~mm}$, de manière à pouvoir travailler avec précision sous fort grossissement. Grâce à cette méthode, le vernis a été aminci progressivement et grâce au contrôle permanent du travail sous loupe binoculaire, le nettoyage a été mené à bien sans porter atteinte ni aux glacis, ni aux rehauts métalliques, ni à la patine de la couche colorée. Cette intervention a permis de retrouver le fond bleu, la palette vive et fraîche du peintre, les détails des architectures, les rehauts d'or, le modelé des visages. Mais si cette méthode permet un travail plus sûr et plus contrôlé que le nettoyage à l'aide de solvants, elle est aussi beaucoup plus lente. Elle a nécessité une cinquantaine de jours pour nettoyer l'œuvre. Cette technique a pu être mise au point au sein des musées russes, qui emploient des restaurateurs d'icônes salariés, tandis que les musées français font travailler des restaurateurs de peinture indépendants. Si le temps passé sur une œuvre ne rentre pas en compte pour un restaurateur salarié, il revêt au contraire une grande importance pour un restaurateur indépendant et pour le conservateur de l'œuvre, car il va se traduire directement en terme de coût de restauration. L'icône du musée du Temps a pu bénéficier de cette technique de restauration car l'intervention s'est faite dans le cadre d'un mémoire de fin d'études et a donc été gratuite, mais il n'est pas sûr que le musée aurait pu assumer le coût d'une pareille restauration. Cette œuvre est donc l'illustration, d'une part, du rapport au temps de l'Église byzantine, et, d'autre part, d'une pratique de la restauration détachée des contraintes de temps et de rentabilité, que nous ne pouvons pas toujours faire nôtre actuellement (fig.2). 
Fig. 2

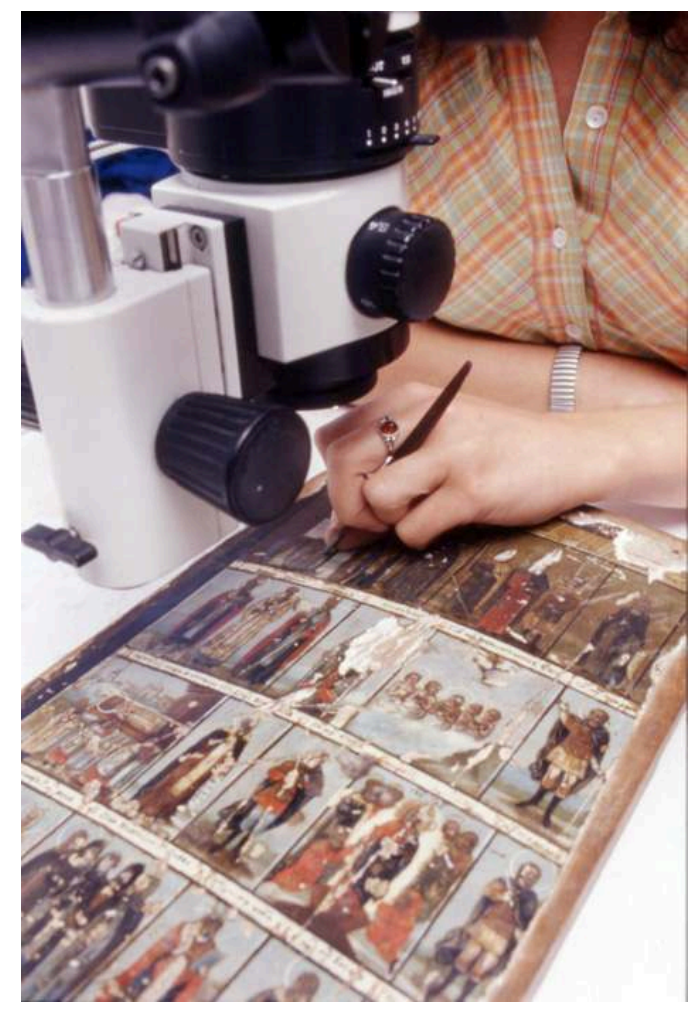

Dégagement sous loupe binoculaire

11 Après le dégagement, la réintégration à l'aquarelle de l'icône a rétabli la lisibilité de l'œuvre tout en respectant l'exigence d'une intervention minimale'. L'œuvre a ensuite été vernie avec un vernis Dammar ${ }^{\circledast}$ mat qui permet d'unifier et de protéger la couche picturale tout en lui conservant son aspect satiné, et le bois ciré à la cire d'abeille.

\section{NOTES}

1. Effectuée au cours de l'année 2001 au sein du département des restaurateurs de l'Institut national du patrimoine.

2. Berelowitsch (A.), Étude et restauration d'une icône-calendrier russe du Musée du Temps, mémoire de fin d'études soutenu au département des restaurateurs de l'Institut national du patrimoine, 2001 (non publié) ; Berelowitsch (A.), «Restauration d'une icône-calendrier russe du musée du Temps à Besançon ", dans Patrimoine des Balkans. Voskopojë sans Frontières 2004, sous la direction de M. Durand, Paris2005, p. 163-174.

3. Patterson-Sevcenko (N.) dans Oxford Dictionary of Byzantium, éd. A. Khazdan, New York, Oxford, 1991 vol. 2, s.v. « Hagiographical illustrations », p. 895-897.

4. Patterson-Sevcenko (N.), Illustrated Manuscripts of the Metaphrastian Menologion, Chicago, 1990.

5. Bobrov (Y. G.), Osnovi ikonografii drevnerousskoï jivopici, Saint-Pétersbourg, 1995.

6. Pour le style des icônes tardives, voir Stuart (J.), Ikons, Londres, 1975. 
7. Masschelein-Kleiner (L.), Liants, vernis et adhésifs anciens, Bruxelles, 1983 ( $3^{\mathrm{e}}$ éd. remise à jour, Bruxelles, 1992).

8. Lelekova (0.), «Problèmes de restauration des icônes russes", Comité de l'ICOM pour la conservation, Dresde, 1990, vol. 2, p. 768-772.

9. Sur la réintégration des icônes, voir Delsaux (N.), «A propos de l'exposition Byzance. Quelques exemples de restauration d'icônes ", La revue du Louvre et des Musées de France, 5-6 (1992), p. 86-93.

\section{AUTEUR}

\section{ALINE BERELOWITSCH}

Aline Berelowitsch est restauratrice indépendante de tableaux, spécialisée en icônes et peintures murales. Après des études de lettres et d'histoire de l'art, elle a obtenu en 2001 le diplôme de l'Institut national du Patrimoine. Elle exerce depuis sa profession aussi bien pour les collections publiques (musées) que pour les particuliers. 